\title{
Review
}

\section{Personal Computer Based Food Engineering Education}

\author{
Murat O. BALABAN ${ }^{1}$ and Saim URAL ${ }^{2}$ \\ ${ }^{1}$ Food Science and Human Nutrition Department, University of Florida, Gainesville, FL 32611, USA \\ ${ }^{2}$ Computer Science Department, Western Washington University, Bellingham, WA 98225, USA
}

Received October 10, 1995

\begin{abstract}
The availability, speed and affordability of personal computers (PCs) enable new possibilities in the education and training of Food Engineers. The use of PCs enables students to grasp complex concepts without getting tangled in computational details, use simulations and ask what-if questions to better understand and visualize phenomena or processes, and eliminate or minimize simplifying assumptions. Internet and World Wide Web make distributed learning possible. Available software are classified into three categories: generalized commercial software such as spreadsheets, semi-specialized but expensive software such as Aspen or MATLAB, and specialized software written by faculty/students in Food Engineering. There is a need to develop educational software by the latter group, since software for small audiences is not economical for commercial software companies. Examples of educational programs are given. A world-wide clearing house for software developed by faculty/students is suggested. The possible mechanisms of such an effort is discussed.
\end{abstract}

Keywords: personal computer, software, education, food engineering

Food Engineering involves the application of engineering principles to agricultural and biological materials for the purpose of supplying safe, abundant, nutritious, palatable and readily available foods at a reasonable cost to the increasing world population. Faced with a decreasing amount of arable land, diminishing natural resources, and concerns about environmental damage, this task is increasingly more difficult for today's student and tomorrow's food engineer. A non-homogeneous and seasonably, geographically, compositionally, and genetically variable raw material makes Food Engineering more challenging than traditional disciplines such as Chemical Engineering. In the U.S., Food Engineering is viewed in conjunction with Chemical and Agricultural Engineering, with generally overlapping subject areas (Bhattacharya et al, 1985). In all three disciplines there are continuing efforts to use new technologies and teaching tools to educate future engineers faced with increasing challenges (Edwards, 1980). Curricula and accreditation requirements are being revised in many countries to update and modernize the education process (Ito, 1974; Jacqmain et al., 1980; Kalman \& Martinez, 1982; Maelkki, 1984; Ojha \& Maheshwari, 1980; Smit \& Wrolstad, 1977). The use of personal computers is a powerful tool in engineering education (Edgar et al., 1985; Finlayson, 1984; Steffe, 1981). The speed of personal computers (PCs) has been doubling every year since their introduction, and their price/performance ratio has been dropping steadily. Many graduate and undergraduate students today have their own PCs, and universities have PC labs available to students.

Advantages of Computers in Food Engineering Education

The old educational practice of relying solely on an instructor in a classroom teaching out of a textbook is coming to an end. Instructors of today have a number of compelling reasons to complement their courses with the use of PCs. These are to: 1) Eliminate or minimize the diluting and obstructing effect of complicated mathematics or calculations to the understanding of the subject matter, 2) Graphically show students the workings and results of a process that they know by theory, 3) Play what-if games to gain a better and deeper understanding of the phenomena or processes, 4) Use new media and instruction methods. Computer aided instruction as a supplementary tool was tried in a food related course in Oregon State University, and was found to be significantly more effective than the "normal" course (Curtis, 1986).

In the past, assignments such as solving a heat transfer problem using successive relaxation method required using pencil and paper. After days of work the student obtained a series of numbers without actually grasping the idea of temperature distribution. The procedure was so effort intensive that the student lost track of the "forest" (the concept of dynamic temperature distribution) for the "trees" (the procedure and effort of calculation). Today, this assignment can be done with a spreadsheet. However, the "programming" and debugging of the spreadsheet is not trivial to students. A computer program can be used to do the calculations and display the results using graphics. Humans value visual input. The graphical output coming from a software greatly enhances the understanding of the subject. Since the tedious calculations are eliminated, students can understand the actual process and the meaning behind all the equations and theoretical discussions. Then, they can change the parameters of the problem and see how results change. Since this happens with a few pushes of a button (assuming that the proper software is available) they will enjoy playing what-if games, and this will help them understand 1) the limits of the process 
Table 1. Types of software available for Food Engineering education.

\begin{tabular}{|c|c|c|c|c|c|}
\hline Software type & Availability & Price & Maintenance and updates & Difficulty of use & Source \\
\hline Spreadsheets & Wide & Medium & Excellent & Medium & Commercial \\
\hline Specialized software & Wide & High & Excellent & High & Commercial \\
\hline Food Engineering specific software & Very narrow & Low & Spotty or non-existent & Low & Developed by faculty or students \\
\hline
\end{tabular}

(i.e., what happens if one parameter is set to a large value, will the process still work?), 2) the effects of various parameters and their relationship (such as, what happens if I change parameter $a$ without changing parameter $b$, what happens if I increase them both at the same time, or if one is increased and the other is decreased, etc.).

\section{Software for Food Engineering Teaching}

Although the prices of the personal computers are falling, and most Food Engineering faculty agree with the advantages of using PCs in teaching, one problem is the availability of software. The software needed for the course work is either not available, or commercially available software is expensive. Specialized software developed by equipment manufacturers such as SimuFerm by Leading Edge, or UPECS (Universal Plant Engineering and Construction System) for dairy plant design and management (Anonymous, 1982, 1987) are generally not available or affordable for teaching. However, there are efforts in the industry to use computers in the training of their engineers (Sumner, 1980; Smith, 1988).

The software needed for education is and should be different from the commercial software. The educational software should not be written for the experienced engineer. The students using the software should be able to get ample help, and should be guided with a number of demonstrations. The software should be user friendly and "user-proof." In a classroom environment we can not afford to use a software that has a steep learning curve, since it will adversely affect the curriculum by spending much time in learning the software rather than using it. Most of the commercial software packages assume that the users have enough time to learn how to use it, and assume that they will be using the same software for a long time, therefore becoming "experts" in it. The fact that there are intensive courses to learn popular software indicates that they are not easy to learn. This is neither possible nor feasible in a classroom environment where students might be using a number of different programs per semester. Even if one class would use one software per semester, this would mean learning between four to seven software per semester for students. Therefore the educational software should be easy to learn and use. The software should allow the use of theoretical limits of parameters rather than practical limits, and may include exercises and review questions.

Another problem with the commercially available software is the price. Although most of the software companies do provide educational or volume discounts, their prices are still beyond the reach of most departments and students. When a department can afford to buy a number of copies of a software it also needs to provide and maintain a number of PCs, and the students face the problem of finding an available computer to do their coursework. A case in point is a class in our department where 600 students need to use a specific software for 3 weeks. Computer labs then become interesting places, to say the least. Students have already recognized the advantages of learning and using computers and they are buying their own PCs. If inexpensive (maybe even free) software can be provided to the students, they will use their own computers at home or dormitories, reducing the pressure on the institutionowned computing resources. They will also maintain their own computers, minimizing the high cost of maintaining a large number of computers at school.

The PC software used in Food Engineering can be classified as: 1) General purpose commercial software such as spreadsheets. These are powerful for simple calculations and/ or graphics, but generally not suited for complex or iterative calculations. The learning curve is easy for simple tasks, but gets more steep as topics such as macros are encountered. 2) Specialized commercial software for general engineering calculations and analysis, such as Aspen, Mathcad, MATLAB, etc. These are powerful programs with steep learning curves and specialized programming languages. They are not inexpensive to be readily available for students. 3) Programs developed by faculty or students for specific sections of a particular course. These programs are generally not known by other departments and universities. They are not maintained and updated in the sense of commercial software (Table 1).

\section{Examples of Available Software}

Spreadsheets The use of spreadsheets in teaching Food Engineering is gaining wide acceptance in Food Engineering (Singh \& Heldman, 1993; Toledo, 1980), as well as other disciplines (Boye et al., 1993). An increasing number of textbooks use spreadsheet examples in semi-automating complex calculations.

Specialized commercial software AutoCAD is a technical drawing program that also has a "SchoolBox" version that includes classroom exercises and additional resources (Anonymous, 1995b). However, the program is not easy to master.

ASPEN is an example of commercially available software package used in the design and analysis of mostly chemical engineering related processes. A number of these processes are applicable to Food Engineering, such as drying, evaporation, distillation, mixing, fluid flow, pipe network design, etc. (Gallier et al., 1980). Although it accomplishes its goals excellently, the software is expensive, and requires that the user learn the intricacies of the input and output system.

CACHE Corp. was founded as a non-profit organization to develop software tools for Chemical Engineering teaching (Karimi \& Reklaitis, 1984). Their programs are not free. More information can be obtained from their World Wide Web site (http://www.che.utexas.edu/cache).

For system modeling problems, continuous system modeling program (CSMP) can be used (Lambert, 1975). The program has its own "language," and is not inexpensive. For 
Table 2. Transient heat conduction example in a cylinder. Written for MATLAB.

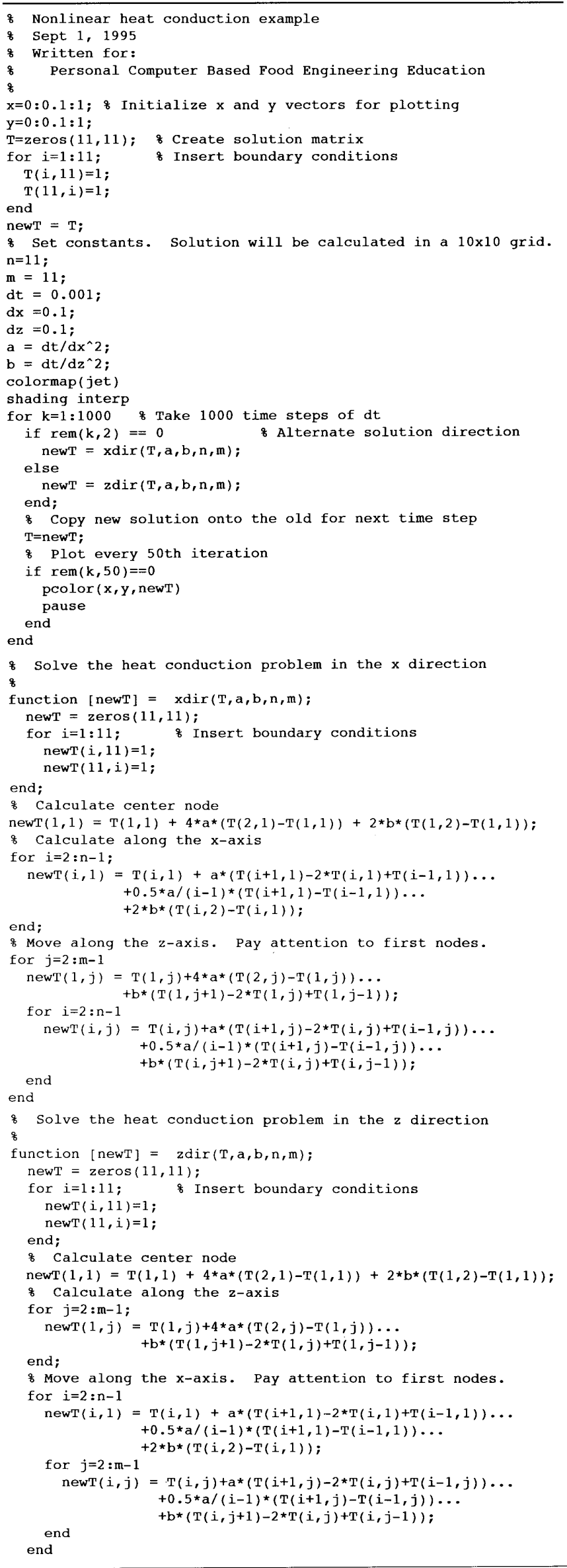

optimization, GRG2 package can be used (Lasdon \& Waren, 1982).

Symbolic mathematics (Ferguson, 1994; Slaughter et al., 1990) and equation solver packages (Young \& Stikeleather, 1991) are used in the instruction of various engineering disciplines.

MATLAB is a program designed to simplify mathematical processes and graphics. A sample MATLAB program to solve transient heat conduction in a finite cylinder is given in Table 2. Although the program is not very long, one needs to have access to MATLAB and to spend time to learn its programming language to write it. MATLAB is one of the easiest software packages an engineering student can learn and use. The built-in matrix and vector operations make programming easier. One can also display color graphics in 2-d and 3-d by calling functions that are part of this software. There are many other specialized packages like MATLAB.

Programs developed by faculty Many disciplines teach thermodynamics (Morse, 1994; Uddin \& Rodriguez, 1992), dynamics (Jacquot et al., 1995), and thermal processes (Schoenhalls \& DeWitt, 1994) with the help of computers. Every Food Engineering Department, program or faculty develop specific software for parts of their curriculum (Adams, 1985; Clark et al., 1984; Gill et al., 1989; Greenfield, 1973; Heldman \& Steffe, 1985; McClellan, 1985; Mulvaney et al., 1985; Okos \& Reklaitis, 1985; Shoemaker \& Russell, 1985). These include, but are not restricted to, real time data acquisition to be used in labs, software to analyze or simulate drying, heat transfer, phase change, thermal processing, psychrometry, etc. We heard of, seen it mentioned in the literature, or seen demonstrations of some of these software, but the major obstacle remains awareness and availability. Since there are no mechanisms to make others aware of the existence of the programs, they are mostly used by the site in which they were developed. Also, even when we are aware of these packages, we do not know how to get them, or are not sure of the effort necessary to run them successfully in our courses, since hardware, operating systems, and other requirements may vary.

Another example of software developed by faculty is the

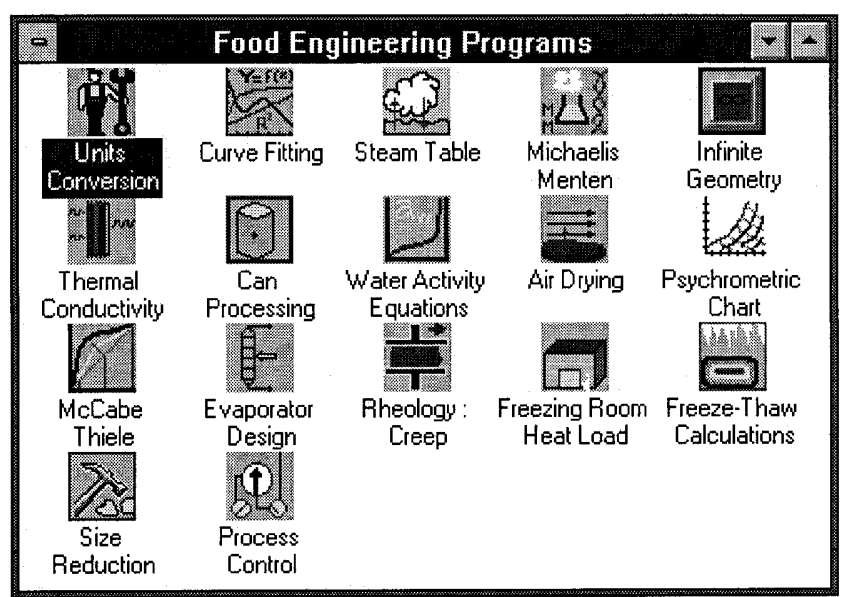

Fig. 1. Windows-based educational programs developed at the University of Florida for Food Engineering. 
Psychrometric Chart Graph

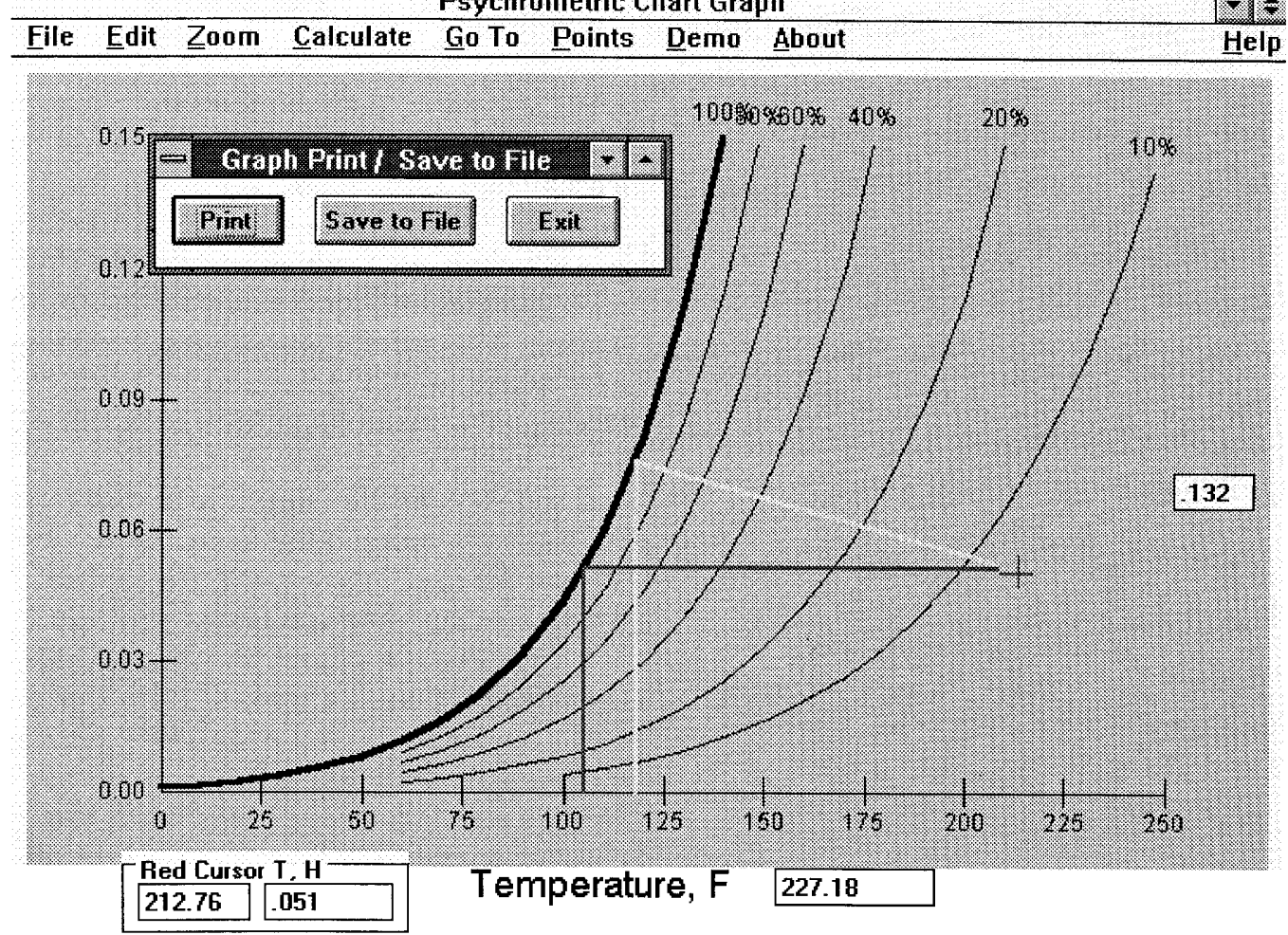

Fig. 2. Demonstration mode of the psychrometric chart program. The screen shows how to locate a point of the chart knowing dew point and wet bulb temperatures.

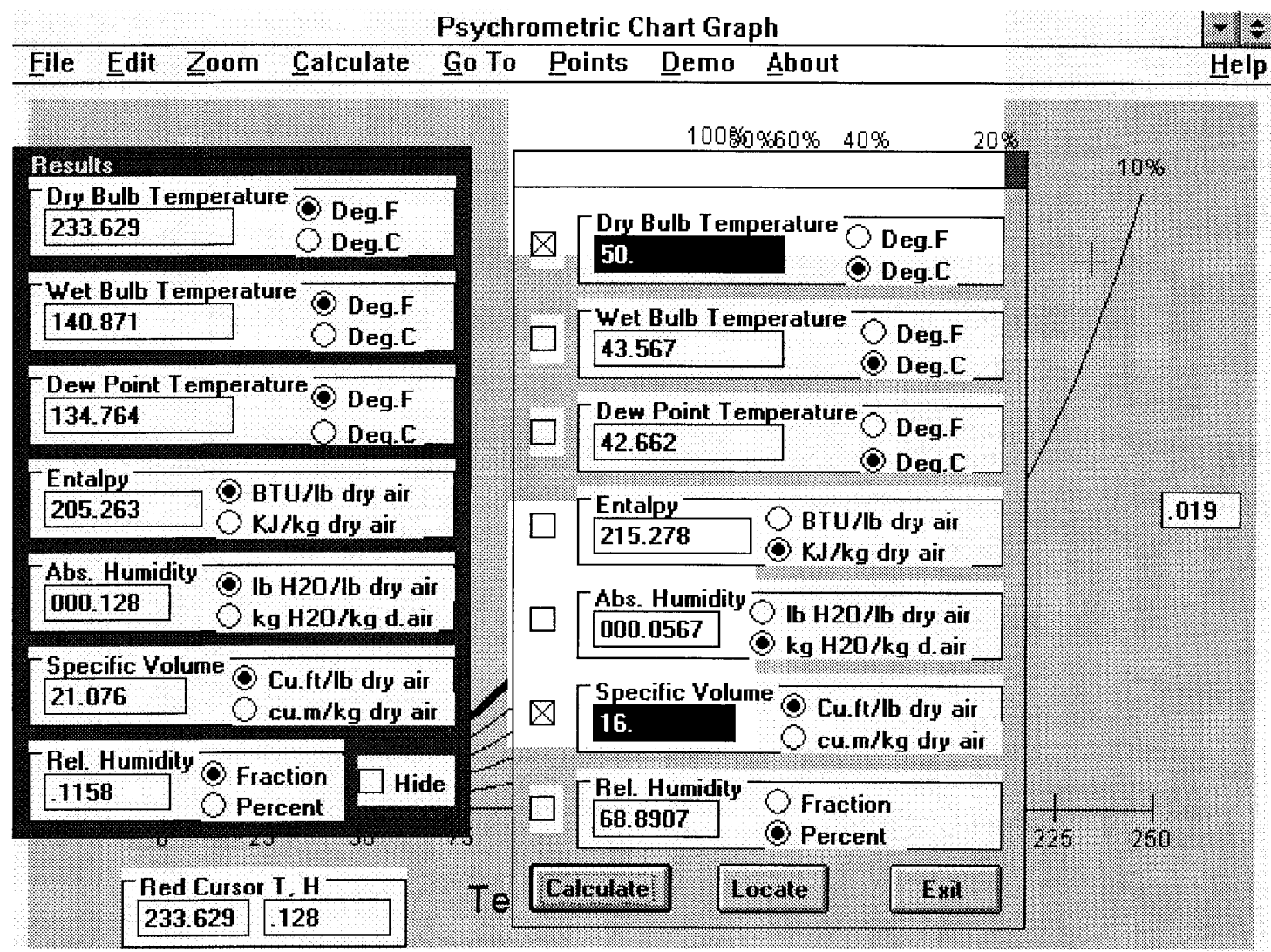

Fig. 3. Calculation of moist air properties with the psychrometric chart program. The screen shows "Results" for the cursor location. Also shown calculation of all properties by knowing any two properties. 


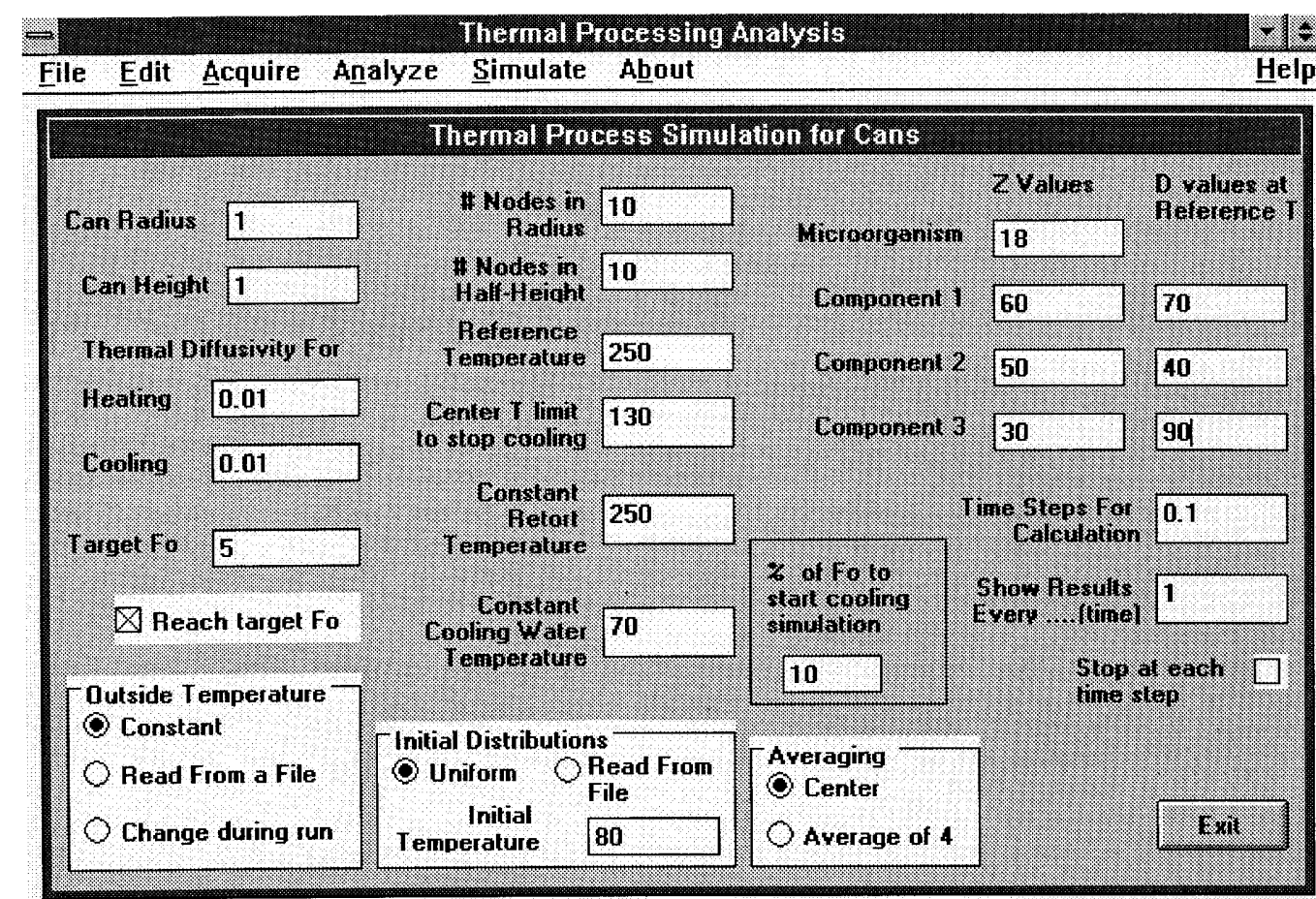

Fig. 4. Simulation screen of the thermal processing program.

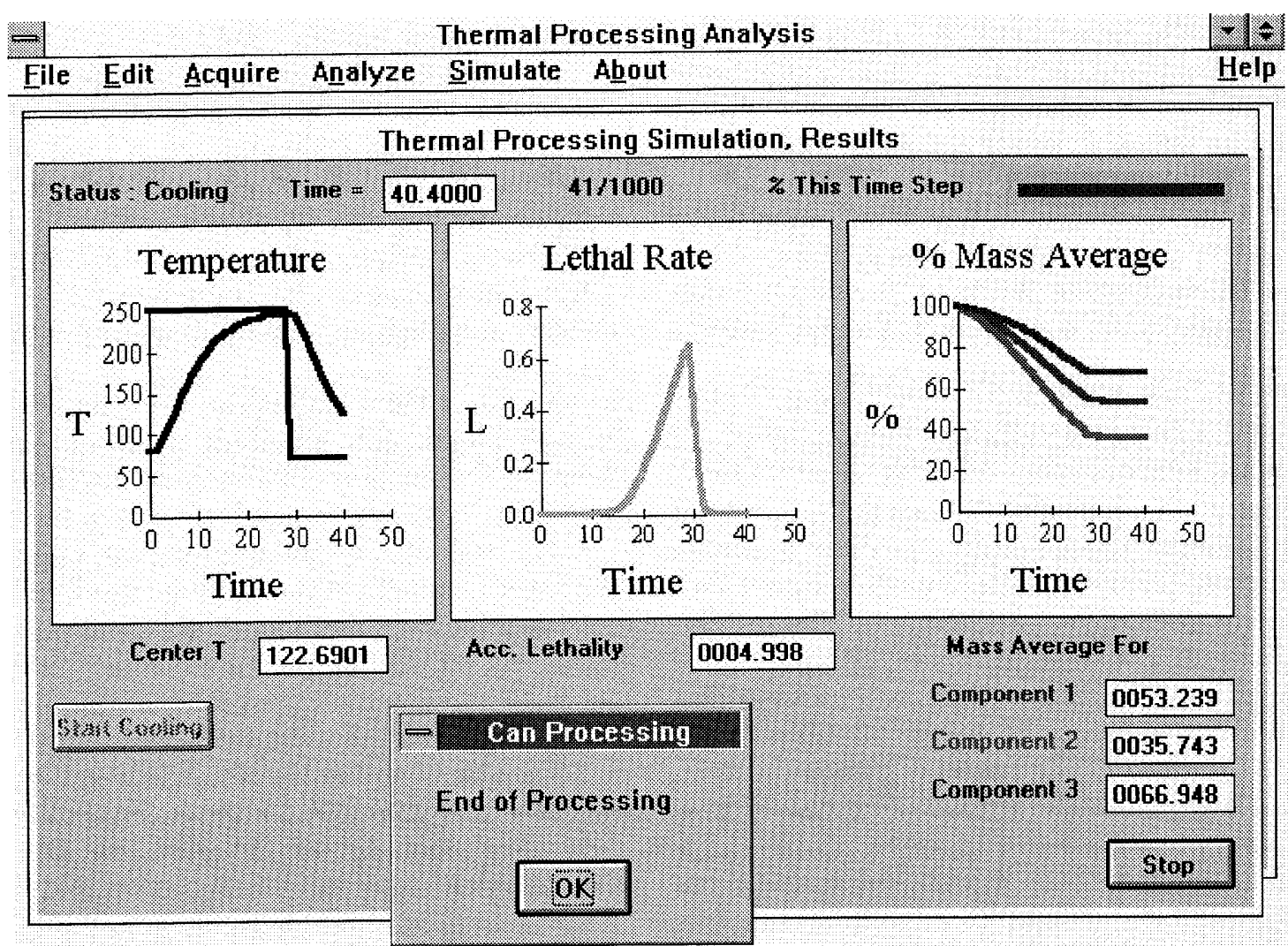

Fig. 5. Running of the thermal processing simulation. Screen shows results of retort and center temperatures, lethal rate, and volume averaged percent retention of components 1,2 and 3 . 


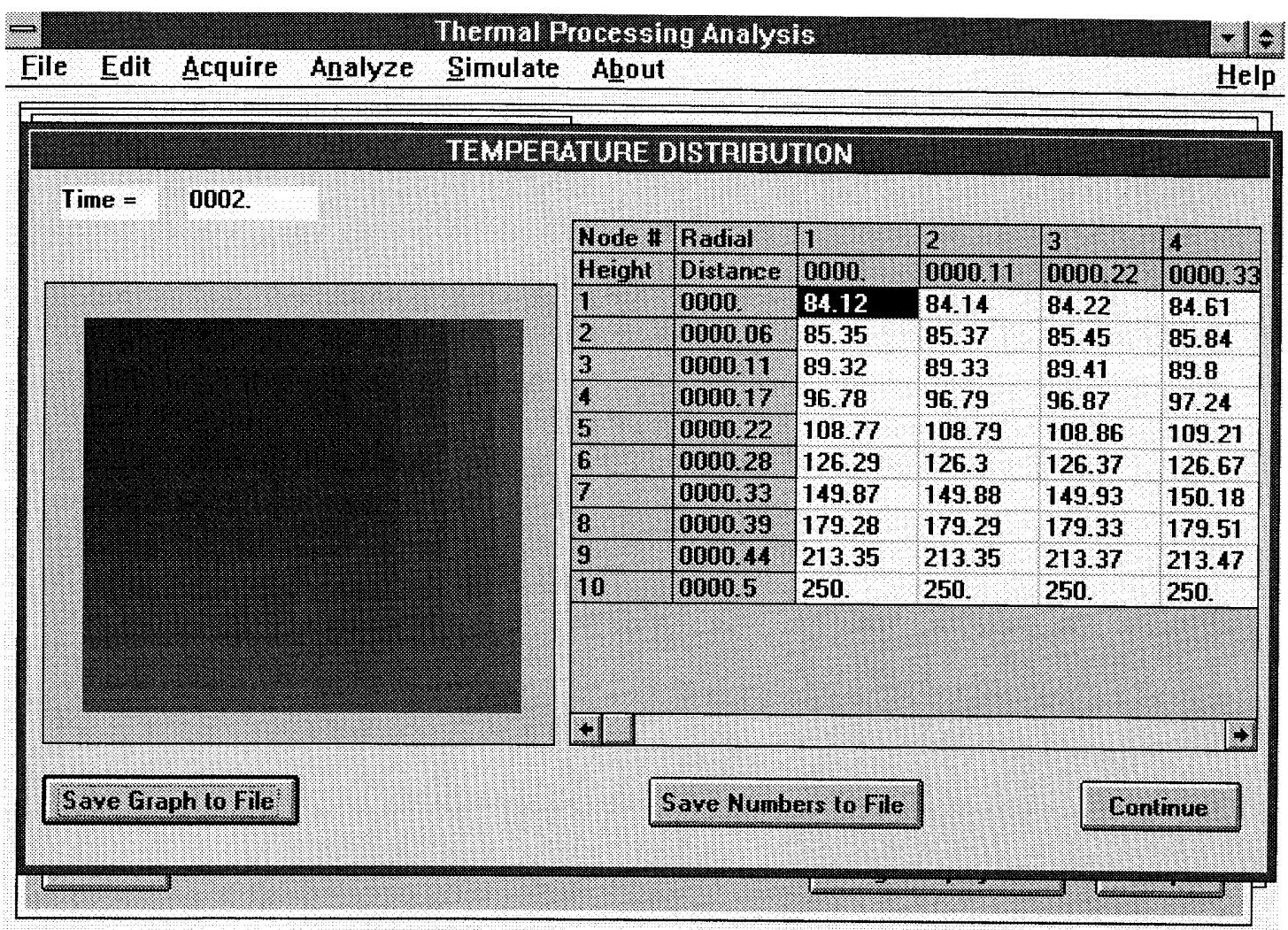

Fig. 6. Running of the thermal processing simulation. Screen shows temperature distribution in the can at 2 min of processing.

RADS (Refrigeration Analysis, Design and Simulation) package, developed by Drs. A.C. Cleland and D.J. Cleland of Massey University, New Zealand. The program has a database of compressor, refrigerant, evaporator etc. specifications that can be used in the design and analysis of refrigeration systems.

There are programs developed in practically every Food Engineering curriculum to analyze thermal processing. Space does not permit to mention all of them. The SterilMate program developed by Dr. A.A. Teixeira at the University of Florida is a good example.

Graphical user interface (GUI) allows for more natural interaction of the program with the user (Houck, 1993), and is being adopted by the industry. In the PC area, Microsoft Windows is the most widely used GUI system. A number of Windows-based Food Engineering programs were developed in our Department at the University of Florida (Fig. 1). Two examples will be given.

Example 1. Psychrometric chart use The teaching of the psychrometric chart is an area where computers can help. The chart is a tool often used in drying calculations. After explaining the origin of the equations describing the chart, and showing how to obtain various properties from it, students move on to solve drying problems. The challenge here is the mechanics of obtaining the values of various parameters. If one is close to the $0^{\circ} \mathrm{C}$ of the chart, it becomes difficult to differentiate between points, necessitating a "zoom in" capability. Also, students sometimes forget how to e.g. locate a point on the chart knowing Dew Point and Wet Bulb
Temperatures. The psychrometric chart program shown in Fig. 2 was written to assist in these areas. The program works in the Windows environment, and has full Cut/Copy/Paste capabilities. Figure 2 shows the "Demo" mode where students are reminded how to locate a point knowing the DewPoint and Wet Bulb Temperature. Many other combinations are also available. Zooming is permitted up to 10 times, facilitating resolution of close points. Figure 3 shows instant calculations of properties (left window) at the cursor location; and the capability of calculating all properties, given any two parameters (right window). The ability to place up to 5 user defined points and to take property differences between these points allows for easy calculation of heating loads, absolute humidity differences, etc. The program has been used at the University Florida for more than 9 years, with great success.

Example 2. Thermal processing of cans This program, also running in Windows environment, is composed of the data acquisition, data analysis and simulation parts. It can gather up to 16 thermocouple readings and calculate accumulated lethalities in real time, it analyzes an existing center temperature-time data and calculates various parameters ( $\mathrm{f}, \mathrm{j}$, effective diffusivity, etc.), and it performs simulated runs of heating and cooling based on many combinations of parameters. Figure 4 shows the property input screen of the simulation part. Note that a desired lethality $F_{\mathrm{o}}$ is entered for the program to find the corresponding steam shut off/cooling water on time. Also note that up to three "components" can be included into the calculations. These can be nutrients or quality factors with known kinetic parameters. Retort temper- 
ature can be constant, or read from a file, or changed manually during the run. In Fig. 5, the results of the run for Fig. 4 are given. Note that volume average percent retention of the three components are calculated. The temperature profile corresponding to the desired lethality is shown. A typical run takes less than a minute, so students can perform many runs and see the various effects of can size, different retort temperatures and profiles, initial product temperatures, etc. on quality. This program has been used successfully in undergraduate food processing classes where students were asked to optimize processes for nutrients. In Fig. 6, the temperature distribution at $2 \mathrm{~min}$ into the processing is shown both graphically and numerically. Nutrient distributions can also be obtained this way. Visual presentation helps students better retain the information, and the effects of the process on various constituents.

\section{Potential Disadvantages}

Computers and educational software are tools, aids, and complements to the instructor (Cox \& Lawrence, 1993). They are not an end by themselves, nor should the results they give blindly trusted. Their proper use for rapid calculations is only after the subject is assimilated by the student. The student should not be made dependent on the computer, but he/she should be able to do the calculations by hand if it becomes necessary. Improper use of computers or software may negate all of their potential contributions to teaching.

\section{Internet and World Wide Web}

The widening use of the Internet allows educators to use it as another medium for information delivery (Bilotta et al, 1995; Blanchard et al., 1994; Campbell et al., 1995). An example of distance learning is the MBA degree offered across the Internet by the Southhampton Institute in England (Anonymous, 1995a). Distributed learning is another concept where resources are decentralized and distributed. The instructor, students and content can be located in different locations, and learning can occur independent of time and place. An example is the World Lecture Hall (http:// wwwhost.cc.utexas.edu/world/instruction/index.html). World wide web (WWW) can be used from basic information delivery to creating virtual learning environments (Dwyer et al. 1995). Other avenues are USENET (Erickson, 1993) and Microsoft Help Compiler to create lessons. The course supplement mode allows faculty to distribute their standard course materials on WWW. Faculty and students meet in the traditional classroom or telecourse, while lecture notes, assignments, syllabus etc are delivered via the WWW (Saltzberg \& Polyson, 1995). This is a good way for faculty to be introduced to WWW. In the virtual classroom mode, WWW becomes the medium for online lectures and instructional materials, interactive multimedia textbooks, one-to-one communications, and access to remote systems. Experiental learning (virtual realities), online testing and assessment become possible (Wright, 1994). In Food Engineering, Dr. R.P. Singh (University of California, Davis) has created a "Web page" for an introductory Food Engineering course with "bullet" topics and simple figures that can be downloaded (http://www.engr.ucdavis.edu/ rpsingh). The bene-

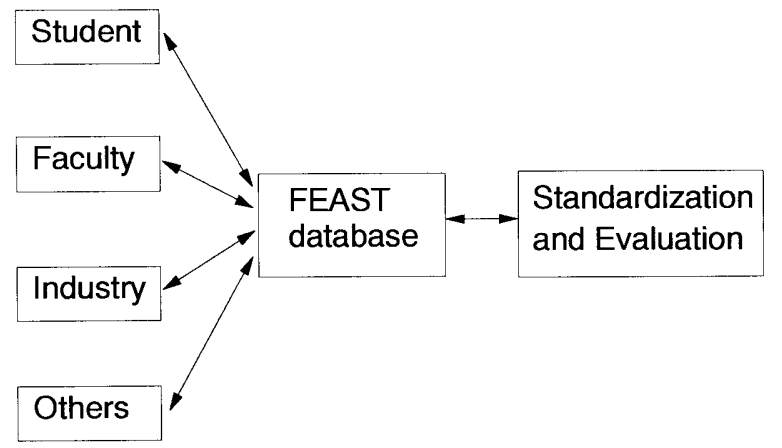

Fig. 7. Flow of programs in the proposed FEAST database and clearing house.

fits of distributed learning are: 1) the environment is student centered and collaborative, 2) convenience, 3) ease of use, 4) quick and easy development of course material, 5) readily available resources, and 6) easy standardized access (Anonymous, 1995a).

\section{Need to Develop and Share Educational Software}

When faculty recognize the importance of using computers in the classroom, they start looking for ways of providing software packages to their students. We believe that faculty are the best source for educational software. They have the expertise in their field, they know what aspects of the subject matter can best be demonstrated with the use of computers, and where and when software would be very useful to their students. However, educational software development by faculty and/or graduate students has been slow and far between, due to several potential impediments. Faculty who are developing software packages need to learn one or more programming languages. Constantly changing hardware and software makes this more difficult and time consuming. The development of good software takes a long time and much effort. Some academic circles do not accept this as scholarly activity. Once the program has been developed, one also needs time to improve and maintain it. This is also a time consuming processes. Over the years, the development of a number of good software packages within academia was for the personal satisfaction and enjoyment of the faculty or graduate student who developed it, and also by the farsightedness of some institutions.

Not all faculty who believe in using computers in teaching can, or should write their own software. They may not have the time or the interest in learning programming languages and developing software. Therefore the idea of using the software developed by someone else is the most cost effective approach, as long as software is easily available.

The idea of sharing programs legally is not new, and has been practiced since the beginning of the computer era. Today, many excellent programs are in the "shareware" market and can be obtained from electronic bulletin boards. We propose to create a database and clearing house of Food Engineering related programs. This may be called FEAST (Food Engineering Aids for Students and Teachers) and may be the depository of the programs developed by the faculty, 
students, and industry (Fig. 7). The database would be housed in two universities, and would be accessed by the Internet. File transfer protocol (FTP) could be used to deposit programs, and after validation, they would be available to everyone. Since more computers are linked together with networks every day, it would be easy to communicate with the database. Anyone who has developed a software and wants to share it with the rest of the world could deposit the software into this database. The FEAST database manager, with the help of a number of faculty and/ or graduate students, may run a number of validation tests, modify the program if necessary, and make it available to anyone free of charge. Validation is an important part of software used for learning (Beattie, 1994). The rules of submitting a software, the validation procedures, the kind of standards that must be imposed upon the software, the programming languages and operating systems that must be used, etc. must be decided and made available to the public before attempting to create such a database.

We are aware that there are many issues to be clarified, such as the type of hardware to be used (IBM-PC vs Mac), operating systems, whether the source code of the programs should also be made available, programming language used, etc. These issue can be resolved with consulations with educators around the world. It is our belief that the source code of these programs should be made available. The advantages of this outweigh the disadvantages. The availability of the source code will, in the long run, help us create and share better programs. If the source code of a program is available, anyone can work to improve it, and resubmit the program to FEAST with proper credits given to the original author. This way we do not have to "reinvent the wheel" and waste valuable time. This will reduce the development cost and time, and will help us create better teaching programs.

\section{Integration of Software Packages to Courses}

Making a number of software packages available in a clearinghouse will not mean much unless these programs are integrated into the courses. The faculty will need assistance in: 1) the usage of available software, 2) techniques of developing educational software, and 3) integration of educational software into specific courses. These can be accomplished by seminars or electronic forums. Annual seminars can be offered by FEAST on 1) and 2) above. New faculty can be introduced to the idea of using personal computers in teaching, and can be assisted in the use of available software. Faculty who have been using these programs can be made aware of new software and the modifications and improvements on existing programs. FEAST could also encourage and provide a forum for faculty who have already integrated PCs in their courses to share their experiences and innovative ways of using them. Textbooks could then use these available programs as tools. Better yet, the availability of these programs would make the development of electronic multimedia "textbooks" easier. The technology enables us to put text, video clips, animation, and educational software on a CD-ROM. All of the materials related to a course can be provided to the students on a CD and they can access the text by using keywords, read the text and see the related anima- tion, print parts of the text, or cut and paste the material to create their own references. A good example would be to read about transient temperature distribution, the equations governing the phenomena, the analytical and numerical methods of solving such a problem, and then to see an animation of this process on demand. These are the kind of books the students of food engineering of today and tomorrow will need.

\section{References}

Adams, J.P. (1985). Statistical analysis in a food science and engineering laboratory course. Food Technol, , 39, 103.

Anonymous. (1982). Process design by computer. Food Eng., 54, 80

Anonymous. (1987). UPECS speeds up design and project management. Dairy Ind. Int., 52, 23.

Anonymous. (1995a). So you want to publish academic software? Syllabus, 9, 14.

Anonymous. (1995b). AutoCAD SchoolBox designed for the classroom. Syllabus, $9,8$.

Beattie, K. (1994). How to avoid inadequate evaluation of software for learning. IFIP Trans. A: Comput. Sci. Technol., A-59, 245.

Bhattacharya, M., Hanna, M.A. and Tatkar, V.K. (1985). Computers assist in teaching food engineering to food science students. Food Technol., (12), 65 .

Bilotta, E., Fiorito, M., Iovane, D., and Pantano, P. (1995). Educational environment using WWW. Comput. Networks ISDN Syst., 27, 905.

Blanchard, S.M., Hale, S.A., Clark, B.P., McCaslin, J.W., Dikeman, J.S. and Pool, T.S. (1994). Biological and biomedical engineering teaching aids on the internet. Annual International Conference of the IEEE Engineering in Medicine and Biology Society-Proceedings. 16, 1420.

Boye, A.J., Williams, P.F. and Soukup, R.J. (1993). Using spreadsheets to teach problem solving in a first year class. IEEE Trans. Educ., $\mathbf{3 6}$, 68

Campbell, J.K., Hurley, S., Jones, S.B. and Stephens, N.M. (1995) Constructing educational courseware using NCSA mosaic and the world-wide web. Comput. Networks ISDN Syst., $27,887$.

Clark, S.L., Joglekar, G., Okos, M.R. and Reklaitis, G.V. (1984). Computer aided design of an energy efficient food processing plant. Paper presented at Annual Meeting, AIChE, San Fransisco, Nov. 1984.

Cox, K.R. and Lawrence, T.J. (1993). Software tools, not computer aided instruction, for effective use of computers in education. IFIP Trans. A: Comput. Sci. Technol., A-35, 63.

Curtis, P.A. (1986). Computer utilization in teaching principles of food science and agriculture in post secondary and extension education. Texas A\&M Univ., College Station, Texas 77843, USA. Diss. Abstr. Int., B, 47, 16.

Dwyer, D., Barbieri, K. and Doerr, H.M. (1995). Creating a virtual classroom for interactive education on the web. Comput. Networks ISDN Sys., 27, 897.

Edgar, T.F., Mah, R.S.H. and Reklaitis, G.V. (1985). Use of computers in chemical engineering education. CEP. Sept. p. 9

Edwards, D.M. (1980). Accreditation and quality assessment of food engineering education programs. ASAE-Paper; No. 80-6524, $18 \mathrm{pp}$

Erickson, C. (1993). USENET as a teaching tool. SIGCSE Bulletin (Association for Computing Machinery, Special Interest Group on Computer Science Education), 25, 43.

Ferguson, G.L. (1994). Symbolic math software use as an engineering teaching aid. Computers in Education Journal/Computers in Education Division of ASEE. 4, 48.

Finlayson, B.A. (1984). The impact of computers on undergraduate education. CEP. Feb. p. 70

Gallier, P.W., Evans, L.B., Bruitt, H.I. and Boston, J.F. (1980) ASPEN: advanced capabilities for modeling and simulation of processors. ACS symposium series 124, p. 293.

Gill, T.A., Thompson, J.W., LeBlanc, G. and Lawrence, R. (1989). Computerized control strategies for a steam retort. J. Food Eng., 10, 
135.

Greenfield, P.F. (1973). An example of simulation applied to food engineering. Food Technol. Austr., 25, 502-503, 505, 507.

Heldman, D.R. and Steffe, J.F. (1985). Educational use of computer models for food freezing processes. Food Technol, 39, 87.

Houck, D. (1993). Point, click, drag, mix. Food Process., 54, 108, 111.

Ito, S. (1974). New techniques in the food industry. Nippon Shokuhin Kogyo Gakkaishi, 21, 196 (in Japanese).

Jacqmain, D., Chominot, A., Sainclivier, M., Arthur, W.R.B., Debling, G.B., Martinez-Suarez, J.M., Gomez, H.C., Weill, L., Scriban, R. Kieninger, A., Geurts van Kessel, M.S.M. and Jowitt, R. (1980) Management training in food industries--higher education in food science and technology in Europe. European Federation of Chemical Engineering, Food Working Party; International Commission for Agricultural \& Food Industries. Brussels, Belgium.

Jacquot, R.G., Smith, D.A., Whitman, D.L. (1995). Software package to enhance the teaching of engineering dynamics. Comput. Appl. Eng. Educ., 3, 21.

Kalman, R.E. and Martinez, J. (1982). Computer applications in food production and agricultural engineering. North Holland Publishing Co. Amsterdam, Netherlands.

Karimi, I. and Reklaitis, G.V. (1984). Intermediate storage in noncontinuous processing. In: Proceedings of Foundations of Computer Aided Design. p. 415. CACHE Corp., Austin, TX.

Lambert, J.R. (1975). The use of CSMP for agricultural engineering problems. Transactions of the ASAE, 18, 573.

Lasdon, L.S. and Waren, A.D. (1982). General reduced gradient software for linearly and non-linearly contained problems. GRG2 users guide. Univ. Texas, Austin.

Maelkki, Y. (1984). Current position in Finland of education and research in food processing. Kem. Kemi, 11, 161.

McClellan, M.R. (1985). An introduction to computer-based process control in a food engineering course. Food Technol, 39, 96.

Morse, J.S. (1994). Restructuring applied thermodynamics: Exploratory thermodynamics. Computers in Engineering, Proceedings of the International Conference and Exhibit $\mathrm{v} / 2$. ASME, New York, NY, USA. p. 879.

Mulvaney, S.J., Houghton, G.E. and Rizvi, S.S.H. (1985). Development of computer-based process control experiments for a food engineering course. Food Technol. 39, 98.

Ojha, T.P. and Maheshwari, R.C. (1980). An effective graduate programme in agricultural engineering with special emphasis on processing and food engineering in India. Indian Dairyman, 32, 135.

Okos, M.R. and Reklaitis, G.V. (1985). Computer aided design and operation of food processes in industry and academia. Food Technol., 39, 107.

Onaral, B. (1990). Engineering courseware: a developer's perspective. Academic Computing. Jan. p. 12.

Schoenhalls, R.J. and DeWitt, D.P. (1994). Integrating fundamentals and industrial applications in a heat transfer course. Proceedings -Frontiers in Education Conference. IEEE, Piscataway, NJ. p. 469.

Shoemaker, C.F. and Russell, G.F. (1985). A course in computer technology for food science students. Food Technol., 39, 92.

Singh, R.P. and Heldman, D.R. (1993). Introduction to Food Engineering. 2nd Ed. Academic Press. New York.

Slaughter, J.M., Zollars, R.L. and Petersen, J.N. (1990). Comparison of PC based mathematics software for undergraduate engineering education. Int. J. Appl. Eng. Educ., 6, 421.

Smit, C.J.B. and Wrolstad, R.E. (1977). Undergraduate curricula in food science and technology. Food Technol, 31, 46.

Smith, R. (1988). An application of computer simulation technology to the training of engineering and production personnel in the biscuit and chocolate industries. 35th Technology Conference 1988. Biscuit, Cake, Chocolate \& Confectionery Alliance, London, UK, Feb. 11-12 p. 61.

Steffe, J.F. (1981). Computers in undergraduate food science education -a survey. Food Technol., Oct. 1981. p. 98.

Sumner, B.P. (1980). Engineering training in the confectionery industry. Confect. Prod., 46, 211-212, 221.

Toledo, R.T. (1980). Fundamentals of Food Process Engineering. AVI Publishing Co. Westport, Connecticut.

Uddin, M. and Rodriguez, E. (1992). Use of computers for thermodynamic problem solving in undergraduate engineering curriculum. Thermodynamics and the Design, Analysis, and Improvement of Energy Systems - 1992 American Society of Mechanical Engineers, Advanced Energy Systems Division (Publication) AES v 27. Publ by ASME, New York, NY, p. 173.

Wright, M.E. (1994). A dynamic exam. Agric. Eng., 75, 16.

Young, J.H. and Stikeleather, L.F. (1991). Use of TK solver in agricultural engineering instruction. Trans. ASAE, 34, 301. 